\title{
Prediction of schema learning by linear regression'
}

A best fitting equation, describing performance in a previous schema learning experiment, quite accurately predicted performance as a function of trials with new Ss, and new patterns having different schemata and different channel capacity. This prediction accuracy was attributed to a pattern generation procedure which permitted manipulation of the relevant population parameter characteristics without altering other characteristics of the patterns.

Attneave \& Arnoult (1956), in commenting on the haphazard pattern selection procedures commonly used in perception studies, pointed out that experimental results can only be generalized with any confidence when patterns are sampled randomly from some well defined population. Strictly speaking, their excellent argument applied only to statistical generalization. A more extensive generalization would apply to samples of patterns randomly selected from populations differing in known parameters. Such generalization, of course, requires a method for manipulating the relevant population parameters without altering other pattern characteristics.

A computer program, VARGUS 7 (Edmonds \& Evans, 1966; Evans, 1964; Evans \& Edmonds, 1966) had been developed which allows quantification of pattern populations in information terms and permits independent manipulation of several population parameters. The patterns from VARGUS 7 are similar in appearance to the "histoform" patterns introduced by Fitts, Weinstein, Rappaport, Anderson, \& Leonard (1956). The VARGUS 7 patterns, however, are produced from a seven element Markov process by mapping the elements into column heights. A schema can be introduced into the patterns by selecting transitional probabilities to favor a most probable sequence (MPS) of column heights; different MPS's constitute different schemata. A population of schemata may also be sampled by forming random permutations of column height sequences. Redundancy is determined by the magnitude of the probabilities associated with each step of the MPS, and stimulus channel capacity in bits per stimulus is manipulated by changing the number of columns in a pattern. All three of these variables can be manipulated independently. Manipulations of schema and redundancy do not affect certain other potentially relevant variables such as the average are.

Edmonds \& Evans (1966) used VARGUS 7 patterns (at a channel capacity of 25.2 bits per stimulus) to investigate schema learning in a situation where the schema had to be abstracted from a set of varying examples. They found that a transfer group receiving prior training with a set of $67 \%$ redundant patterns reproduced different $67 \%$ redundant patterns, containing the same MPS, significantly better than a control group receiving prior training with $0 \%$ redundant patterns (having no schema). This result indicated that $\mathrm{Ss}$ in the transfer group abstracted the MPS in the set of patterns and used it to reproduce individual patterns. Furthermore, schema learning was found to occur quite rapidly, without knowledge of results, and apparently without Ss awareness.

The purpose of the present study was to determine how accurately the results of the above experiment could be used to predict performance as a function of trials in a new experiment with the same task, but with new Ss and different patterns. The patterns in the present experiment differed from those in the above experiment in two ways. First, they had smaller channel capacity (12 columns as compared to 30 columns in the previous study). Second, they represented four different schemata instead of one. These schemata were randomly drawn from the population of possible schemata. Thus the present experiment was intended to determine whether the results of the previous experiment could be generalized (1) to new Ss, (2) to new schemata, and (3) to new levels of channel capacity. Methed

Subjects. The Ss were 28 undergraduates enrolled in psychology courses at Texas Christian University. They were randomly assigned to the four schema groups of seven Ss each.

Patterns. Each of four different MPS's, designated pattern set PS1, PS2, PS3, and PS4, was used in a Markov process to produce 12 column $67 \%$ redundant patterns (yielding a channel capacity of 10.07 bits per stimulus).

Procedure. Each of the four schema groups reproduced 15 patterns from the corresponding pattern set. A projector exposed each pattern onto a screen for $\mathbf{2 0}$ sec. The Ss were run in groups of four or less. After each pattern exposure, Ss turned to the appropriate sheet in a mimeographed answer booklet where the pattern was printed with three columns omitted. The Ss were allowed $20 \mathrm{sec}$. to draw the three omitted columns in each pattern. The presentation order of the patterns was randomized for each group of Ss. The intertrial interval was $20 \mathrm{sec}$. No knowledge of results was given.

\section{Results and Discussion}

An analysis of variance based on the last five trials on each task was used to evaluate performance dif- 


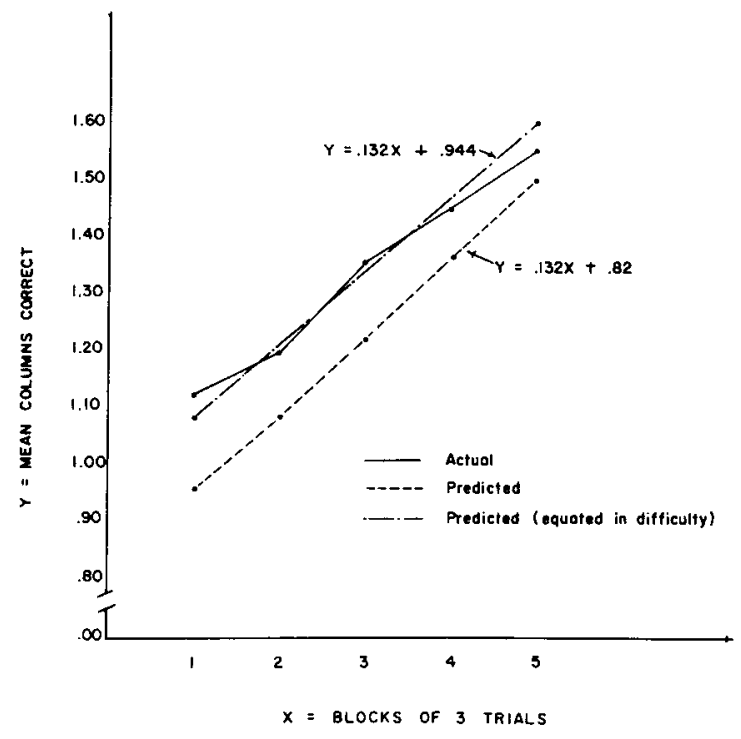

Fig. 1. Actual and predicted data for schema groups.

ferences between the schema groups. This comparison was nonsignificant, indicating that MPS's drawn under the given sampling rules did not differ significantly in difficulty. Data representing all four MPS's were then combined.

In the previous experiment (Edmonds \& Evans, 1966) trend analyses indicated that performance as a function of trials (in the first 15 trials) was very well described by a linear trend. When trials were grouped in fivetrial blocks, the equation $\mathrm{Y}=.22 \mathrm{X}+.66$ was derived from the first three blocks. For the present study, the slope of this equation was adjusted by a factor of .60 (i.e., .60X.22=.132) because trials were plotted in blocks of three each rather than in blocks of five. This adjustment changed the intercept of the predictive equation from .66 to .83 . Both the predicted results and the actual data are shown in Fig. 1. The ratio of the square of the standard error of estimate to the total variance of the means was .55 , indicating that $45 \%$ of the variance of the means was accounted for by the predictive equation $\mathrm{Y}=.132 \mathrm{X}+.83$. The predictive equation underestimated performance, presumably because more information had to be transmitted with 30 column patterns than with 12 column patterns. To provide a better prediction, the intercept of the predictive equation was modified so that the slope passed through the mean of the actual data. This procedure adjusted the prediction for level of difficulty while retaining the predicted slope or rate of improvement in performance. The ratio of the square of the standard error of estimate to the total variance of the actual means was .04, indicating that $96 \%$ of the variance was accounted for by the adjusted predictive equation $\mathrm{Y}=.132 \mathrm{X}+.944$. This equation provided a minimal estimate of unpredicted variance, but should be a better indication of predictive power than the equation which ignored differences in pattern difficulty.

The best fit line computed from the actual data had the form $Y=.147 X+.90$. The slope of this best fit line (.147) did not differ significantly from the slope (.132) of the predictive equation obtained in the previous experiment. Since the slope presumably reflects rate of schema learning, this result suggests that schema learning is not importantly affected by changes in stimulus channel capacity within the range covered by the two experiments. If so, only the overall level of performance, reflected by the intercept, is specific to channel capacity. A study to find a predictive equation relating intercept to channel capacity might thus allow accurate predictions in a large set of pattern populations.

The effect of redundancy on performance in this task is yet to be explored. Since redundancy measures the extent to which patterns adhere to the schema, a decrease in redundancy might be expected to retard schema learning and thus diminish the slope of the line, possibly without altering the intercept. Further research, with redundancy as a variable, should seek a functional relation between redundancy and the parameters of the performance curve.

The present research demonstrates that appropriate pattern generation methods allow generalization of results in a reproduction task to new $S s$, and to new populations of patterns differing in schema and channel capacity. This generalization was made possible by a pattern generation process which allowed manipulation of schema and channel capacity without altering other pattern characteristics.

\section{References}

Attneave, F., \& Arnoult, M. D. The quantitative study of shape and pattern perception.. Psychol. Bull., 1956, 53, 452-471.

Edmonds, E. M., \& Evans, S. H. Schema learning without a prototype. Psychon. Sci., 1966, 5, 247-248.

Evans, S. H. A model for perceptual category formation. Unpublished doctoral dissertation. Texas Christian University, 1964.

Evans, S. H., \& Edmonds, E. M. Schema discrimination as a function of training. Psychon. Sci., 1966, 5, 303-304.

Fitts, P. M., Rappaport, M., Weinstein, M., Anderson, N., \& Leonard, J. A. Stimulus correlates of visual pattern perception: A probability approach. J. exp. Psychol., 1956, 51, 1-11.

\section{Note}

1. This study is based upon a dissertation submitted by the first author to the Graduate School of Texas Christian University in partial fulfillment of the requirements for the $\mathrm{Ph.D}$. degree. 